\section{Prevalencia de consumo de drogas en estudiantes universitarios que cursan primer y cuarto año}

\author{
GLADYS MORALES I. ${ }^{1, \mathrm{a}}$, CARLOS DEL VALLE R. ${ }^{2, \mathrm{~b}}$, CARLOS BELMAR M.,c, \\ YASNA ORELLANA Z. ${ }^{4, d}$, ALVARO SOTO V. ${ }^{5}$, DANIZA IVANOVIC M. ${ }^{4, a}$
}

\section{Prevalence of tabacco, alcohol and marijuana consumption among university students}

Background: University students are especially vulnerable towards substance abuse Aim: To describe and compare drug consumption in students of a Chilean university who attended first and fourth year of studies, according to gender and faculty. Material and Methods: A representative, stratified and proportional sample of 305 students was randomly chosen by faculty, career, grade and gender during 2009. The consumption of alcohol, tobacco and illicit drugs was evaluated using an anonymous survey. Results: Students from health care faculties had the lower prevalence of consumption of tobacco, alcohol, tobacco-alcohol and marijuana, during the first and fourth year. Education area and social sciences faculties had the highest prevalence of consumption. Fourth year students had higher rates of consumption than their first year counterparts. Females had significantly lower rates of alcohol and marijuana consumption. Conclusions: Alcohol, tobacco and marijuana consumption was higher among students from education and social sciences faculties and those attending the fourth year of studies.

(Rev Med Chile 2011; 139: 1573-1580).

Key words: Health Promotion; Lifestyle; Students; Substance related disorders.

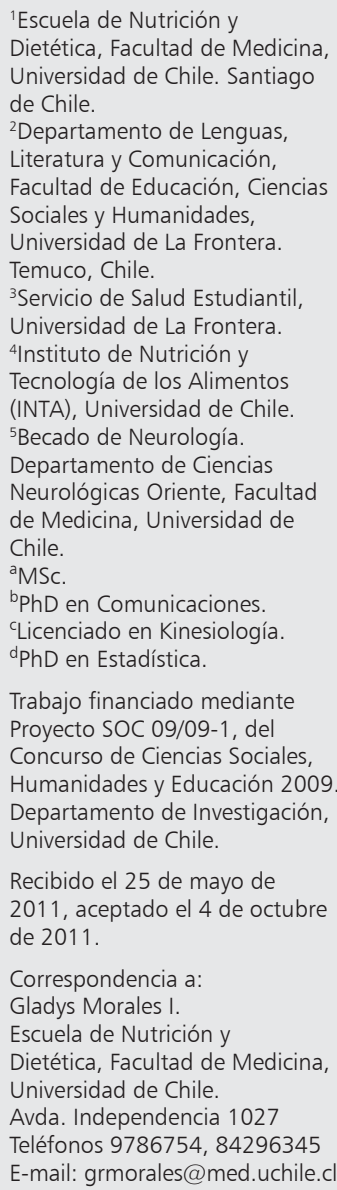

E 1 consumo de tabaco origina más de $90 \%$ de los cánceres de pulmón en hombres, $70 \%$ en mujeres, y se relaciona con $56 \%$ a $80 \%$ de las enfermedades respiratorias crónicas y $22 \%$ de las enfermedades cardiovasculares (sin contemplar el tabaquismo pasivo). A su vez, el consumo excesivo de alcohol es responsable de 3,2\% de las muertes y $4 \%$ de los años de vida ajustados según discapacidad (AVAD) en la población mundial y se relaciona con más de 60 enfermedades y traumatismos, aparte de sus efectos psicosociales en individuos, familias y comunidad ${ }^{1}$.

El consumo de tabaco y alcohol, en la población de 17 a 24 años es mayor en hombres que en mujeres, según la II Encuesta Nacional de Salud 2009, presentándose $44,2 \%$ y $37,1 \%$ respectivamente, en la categoría de "fumadores actuales", situación muy similar a la encontrada con respecto al consumo de alcohol ${ }^{2}$. Además, de acuerdo al estudio realizado por el Consejo Nacional para Estupefacientes (CONACE) en 2008, en estudiantes de universidades e institutos de educación superior, con respecto a las prevalencias de consumo de tabaco, alcohol y marihuana se observó la misma situación $^{3}$. Estos resultados coinciden con estudios realizados en países europeos ${ }^{4}$.

De acuerdo a varias publicaciones, los estudiantes universitarios se encuentran en una etapa vulnerable, caracterizada por un alto consumo de tabaco y alcohol, que se agudiza en cursos superio$\mathrm{res}^{5}$. Esto ha sido mayormente estudiado en estudiantes de medicina ${ }^{6,7}$. Por lo tanto, la importancia del rol que cumplen las instituciones de educación superior y en particular las universidades, como agentes promotores de salud y bienestar, no se ha analizado suficientemente ${ }^{8}$. 
El presente trabajo se realizó con el objetivo de describir y comparar las prevalencias en el consumo de tabaco, alcohol y drogas ilícitas, en estudiantes de una universidad chilena que cursaban primer y cuarto año de sus respectivas carreras. Otro objetivo del estudio fue determinar la asociación entre el consumo de tabaco de los estudiantes con el hábito de fumar de sus padres. Además, identificar el efecto de la restricción del consumo de tabaco y alcohol, impuesta en las dependencias de la universidad, en los hábitos de consumo de los estudiantes.

\section{Material y Métodos}

\section{Diseño del estudio}

Estudio de corte transversal, analítico y comparativo.

\section{Definición de la población y de la muestra}

La población estuvo representada por 2.265 estudiantes de una universidad chilena, de los cuales 1.373 ingresaban a primero y 892 cursaban cuarto año, matriculados en el año 2009, de las cuatro facultades de la universidad, en las áreas de salud; educación y ciencias sociales; ingeniería y ciencias agropecuarias. En total, se consideraron 26 de un total de 34 carreras.

Se seleccionó una muestra aleatoria, representativa, estratificada y proporcional según facultad, carrera, curso y género, correspondiendo a 305 estudiantes. El criterio de inclusión para la elección de las carreras fue que éstas tuvieran estudiantes matriculados en primero y cuarto año. De acuerdo a la distribución de la muestra según curso y facultad a la que pertenecían, se encontró que el mayor porcentaje de la muestra $(33,7 \%)$, pertenecía a la facultad del área de ingeniería (FAI), luego la facultad del área de educación y ciencias sociales (FAECS) (31,5\%), facultad del área de salud (FAS) $(30,8 \%)$ y facultad del área de las ciencias agropecuarias ( FACA) (4\%). En esta última, sólo la carrera de Agronomía, cumplía con los requisitos de inclusión.

En relación a las características generales del grupo estudiado, se encontró que la edad promedio de los estudiantes correspondió a 19,4 y 22,5 años, en primero y cuarto año, respectivamente. De acuerdo a la distribución de los estudiantes según facultad y género, se observó que la FAI presentó el mayor porcentaje de varones (64\%) y la FAS mostró el mayor porcentaje de mujeres (66\%). Además tanto la FAECS como la FACA presentaron similares porcentajes de estudiantes de ambos géneros $(\mathrm{P}<0,001)$.

Este estudio fue aprobado por el Comité de Ética en Estudios Humanos de la universidad. El consentimiento informado se obtuvo de acuerdo a las Normas de Experimentación en Humanos, Código de Ética de la Asociación Médica Mundial (Declaración de Helsinki) ${ }^{9}$. El estudio en terreno se efectuó en el mes de agosto de 2009. ilícitas.

Medición del tabaquismo, alcohol y drogas

El instrumento utilizado correspondió a la Encuesta CONACE 2008 ${ }^{3}$. Esta encuesta fue entregada a cada estudiante para su autoaplicación, solicitando colaboración voluntaria y firma de consentimiento informado, asegurándoles anonimato y confidencialidad. La información solicitada incluyó consumo de alcohol, tabaco y drogas ilícitas (marihuana, cocaína y pasta base). Además de información sobre la edad, género, facultad y curso al que pertenecía cada estudiante.

Se definió como consumidor de tabaco al estudiante que fumaba al menos un cigarrillo en el último mes. Esto fue medido en prevalencia mensual y número de cigarrillos. Se consideró como consumidor de alcohol, al estudiante que consumía al menos un vaso de alcohol en el último mes. Esto se expresó en prevalencia mensual y gramos de alcohol a la semana. Finalmente, se definió como consumidor de drogas ilícitas, al estudiante que hubiera consumido drogas al menos una vez durante el último mes.

La aplicación de la encuesta fue supervisada por el equipo investigador.

\section{Análisis estadístico}

El análisis estadístico se efectuó a través del software estadístico STATA 10.0. Se utilizaron pruebas estadísticas no paramétricas de asociación (test chi-cuadrado) y de comparación (test de KruskalWallis), en el caso que las variables no presentaran una distribución normal. El nivel de significación utilizado correspondió al 5\% ${ }^{10}$.

\section{Resultados}

La Tabla 1 muestra que existen diferencias significativas según facultad, con respecto al consumo de tabaco. El mayor porcentaje de estudiantes que 
Tabla 1. Prevalencia de consumo de tabaco, alcohol y marihuana durante el último mes, según la facultad a la que pertenecen los estudiantes universitarios

\begin{tabular}{|c|c|c|c|c|c|c|c|c|c|c|c|}
\hline \multirow[t]{2}{*}{ Variables } & \multicolumn{2}{|c|}{ FAI } & \multicolumn{2}{|c|}{ FAS } & \multicolumn{2}{|c|}{ FAECS } & \multicolumn{2}{|c|}{ FACA } & \multicolumn{2}{|c|}{ Total } & \multirow[t]{2}{*}{ valor $\mathbf{p}$} \\
\hline & $\mathbf{n}$ & $\%$ & $\mathbf{n}$ & $\%$ & $\mathbf{n}$ & $\%$ & $\mathbf{n}$ & $\%$ & $\mathbf{n}$ & $\%$ & \\
\hline \multicolumn{12}{|c|}{ Consumo de tabaco } \\
\hline Sí & 40 & 38,8 & 16 & 17,0 & 40 & 41,7 & 4 & 33,3 & 100 & 32,8 & $<0,001$ \\
\hline No & 63 & 61,2 & 78 & 83,0 & 56 & 58,3 & 8 & 66,7 & 205 & 67,2 & \\
\hline \multicolumn{12}{|c|}{ Consumo de alcohol } \\
\hline Sí & 72 & 70,0 & 47 & 50,0 & 65 & 67,7 & 8 & 66,7 & 192 & 63,0 & $<0,05$ \\
\hline No & 31 & 30,0 & 47 & 50,0 & 31 & 32,3 & 4 & 33,3 & 113 & 37,0 & \\
\hline \multicolumn{12}{|c|}{ Consumo alcohol y tabaco } \\
\hline Sí & 40 & 38,8 & 15 & 16,0 & 38 & 39,6 & 4 & 33,3 & 97 & 31,8 & $<0,001$ \\
\hline No & 63 & 61,2 & 79 & 84,0 & 58 & 60,4 & 8 & 66,7 & 208 & 38,2 & \\
\hline \multicolumn{12}{|c|}{ Consumo de marihuana } \\
\hline Sí & 8 & 7,8 & 1 & 1,0 & 13 & 13,5 & 0 & 0 & 22 & 7,2 & $<0,01$ \\
\hline No & 95 & 92,2 & 93 & 99,0 & 83 & 86,5 & 12 & 100 & 283 & 92,8 & \\
\hline
\end{tabular}

FAl: Facultad del área de Ingeniería. FAS: Facultad del área de la Salud. FAECS: Facultad del área de la Educación y Ciencias Sociales. FACA: Facultad del área de las Ciencias Agropecuarias. Nota. Se aplicó el Test $\chi^{2}$.

fumó el último mes pertenecían a la FAECS. En relación al consumo de alcohol, la FAI presentó la mayor prevalencia. En cuanto al consumo de alcohol y tabaco fue significativamente mayor en la FAECS, seguida por la FAI. Situación muy similar se observa en las prevalencias de consumo de marihuana. Sin embargo, es importante destacar que la FAS es la que presentó las menores prevalencias en el consumo de tabaco, alcohol y tabaco-alcohol. En la muestra en estudio, ningún estudiante reportó haber consumido cocaína, pasta base u otras drogas ilícitas.

En la Tabla 2 se destaca que los estudiantes de cuarto año, en comparación con los de primero, presentaron un mayor consumo de alcohol $(P<$ 0,05).

En cuanto a la edad de inicio del consumo de alcohol, sólo se observaron diferencias significativas al comparar las medianas de los estudiantes de primer año (16 años), con los de cuarto año (17 años) $(P<0,01)$. Sin embargo, los gramos consumidos por los estudiantes de primer año, son mayores que lo observado en los estudiantes de cuarto año, sin diferencias significativas (Tabla 3 ).

La Tabla 4 muestra que los estudiantes de género femenino, en comparación con los de género masculino presentaron un menor consumo de tabaco, alcohol $(P<0,001)$, tabaco-alcohol y marihuana $(P<0,05)$.

Es importante destacar que las medianas del consumo de gramos de alcohol a la semana son muy similares entre estudiantes mujeres $(15,5 \mathrm{~g})$ y hombres (15,0 g).

Al comparar el porcentaje de las estudiantes con respecto al consumo de alcohol según curso, se observa que existe un mayor porcentaje de mujeres que consume alcohol en cuarto año $(61,5 \%)$, en comparación con las que cursan primero $(46,7 \%)$ $(P=0,067)$.

Además es importante mencionar que, al comparar el consumo de tabaco de acuerdo al género y el año que cursaban los estudiantes, se observó que las mujeres de cuarto año presentaron mayores porcentaje de consumo (51\%), en comparación con los hombres del mismo curso $(39,6 \%)$, aunque sin diferencias significativas $(P=0,349)$. Sin embargo, al comparar las mujeres fumadoras de primero vs cuarto año, sólo se observaron diferencias significativas en la FAECS (50\% y 70\%, respectivamente) $(P<0,05)$.

La Figura 1 muestra el porcentaje de estudiantes fumadores y no fumadores, según la respuesta a la pregunta ¿alguien fuma en tu hogar? Se observa que el porcentaje de estudiantes que no fumaba se 
Tabla 2. Prevalencia de consumo de tabaco, alcohol y marihuana durante el último mes, según el año que cursan los estudiantes universitarios

\begin{tabular}{|c|c|c|c|c|c|c|c|}
\hline \multirow[t]{2}{*}{ Variables } & \multicolumn{2}{|c|}{$1^{\circ}$ año } & \multicolumn{2}{|c|}{$4^{\circ}$ año } & \multicolumn{2}{|c|}{ Total } & \multirow[t]{2}{*}{ valor $p$} \\
\hline & $\mathbf{n}$ & $\%$ & $\mathbf{n}$ & $\%$ & $\mathbf{n}$ & $\%$ & \\
\hline \multicolumn{8}{|c|}{ Consumo de tabaco } \\
\hline Sí & 56 & 30,6 & 44 & 36,0 & 100 & 32,8 & NS \\
\hline No & 127 & 69,4 & 78 & 64,0 & 205 & 67,2 & \\
\hline \multicolumn{8}{|c|}{ Consumo de alcohol } \\
\hline Sí & 106 & 58,0 & 86 & 70,5 & 192 & 63,0 & $<0,05$ \\
\hline No & 77 & 42,0 & 36 & 29,5 & 113 & 37,0 & \\
\hline \multicolumn{8}{|c|}{ Consumo de tabaco y alcohol } \\
\hline Sí & 55 & 30,0 & 42 & 34,4 & 97 & 31,8 & NS \\
\hline No & 128 & 70,0 & 80 & 65,6 & 208 & 68,2 & \\
\hline \multicolumn{8}{|c|}{ Consumo de marihuana } \\
\hline Sí & 13 & 7,1 & 9 & 7,4 & 22 & 7,2 & NS \\
\hline No & 170 & 92,9 & 113 & 92,6 & 283 & 92,8 & \\
\hline
\end{tabular}

NS: no significativo. Nota. Se aplicó el Test $\chi^{2}$.

Tabla 3. Comparación de las mediana de edad de inicio del consumo de tabaco, alcohol y marihuana en el último mes, según año que cursan los estudiantes universitarios

\begin{tabular}{|c|c|c|c|c|}
\hline \multicolumn{2}{|l|}{ Variables } & $\begin{array}{l}1^{\circ} \text { año } \\
\text { Md (p25-p75) }\end{array}$ & $\begin{array}{l}4^{\circ} a n ̃ o \\
\text { Md (p25-p75) }\end{array}$ & valor $p$ \\
\hline Consumo de tabaco & $(n=100)$ & $\mathrm{n}=56$ & $\mathrm{n}=44$ & \\
\hline \multicolumn{2}{|l|}{ Edad de inicio } & $16(14-17)$ & $16(15-18)$ & NS \\
\hline \multicolumn{2}{|l|}{$N^{0}$ de veces a la semana } & $5(4-7)$ & $7(3-15)$ & NS \\
\hline \multicolumn{2}{|c|}{$N^{\circ}$ de cigarrillos a la semana } & $10(5-20)$ & $10(5-20)$ & NS \\
\hline Consumo de alcohol & $(n=192)$ & $n=106$ & $\mathrm{n}=86$ & \\
\hline Edad de inicio & & $16(15-17)$ & $17(15-18)$ & $<0,01$ \\
\hline Gramos a la semana & & $16(9-30)$ & $14,5(12-22,5)$ & NS \\
\hline Consumo de marihuana & $(n=22)$ & $n=13$ & $\mathrm{n}=9$ & \\
\hline Edad de inicio & & $18(16-18)$ & $17(17-18)$ & NS \\
\hline
\end{tabular}

Md: mediana; p25: percentil 25; p75: percentil 75; NS: no significativo. Nota. Comparación de medianas a través del Test de Kruskal Wallis.

asoció significativamente con el hecho que nadie fumaba en su hogar $(P<0,001)$.

Es importante mencionar que al preguntarle a los estudiantes fumadores si la restricción al interior de la universidad, había disminuido su consumo, sólo $39 \%$ señaló que "sî" y, al preguntarles a los estudiantes bebedores de alcohol, sólo
$38 \%$ señaló que la restricción en la universidad había hecho disminuir su consumo.

\section{Discusión}

Los estudiantes de la FAS fueron los que presentaron las menores prevalencias del consumo 
Tabla 4. Prevalencia de consumo de tabaco, alcohol y marihuana durante el último mes, según el género de los estudiantes universitarios

\begin{tabular}{|c|c|c|c|c|c|c|c|}
\hline \multirow[t]{2}{*}{ Variables } & \multicolumn{2}{|c|}{ Femenino } & \multicolumn{2}{|c|}{ Masculino } & \multicolumn{2}{|c|}{ Total } & \multirow[t]{2}{*}{ valor $p$} \\
\hline & $\mathbf{n}$ & $\%$ & $\mathbf{n}$ & $\%$ & $\mathbf{n}$ & $\%$ & \\
\hline \multicolumn{8}{|c|}{ Consumo de tabaco } \\
\hline Sí & 47 & 30,0 & 53 & 35,8 & 100 & 32,8 & NS \\
\hline No & 110 & 70,0 & 95 & 64,2 & 205 & 67,2 & \\
\hline \multicolumn{8}{|c|}{ Consumo de alcohol } \\
\hline Sí & 83 & 52,9 & 109 & 73,6 & 192 & 63,0 & $<0,0001$ \\
\hline No & 74 & 47,1 & 39 & 26,4 & 113 & 37,0 & \\
\hline \multicolumn{8}{|c|}{ Consumo de tabaco y alcohol } \\
\hline Sí & 45 & 28,7 & 52 & 35,1 & 97 & 31,8 & NS \\
\hline No & 112 & 71,3 & 96 & 64,9 & 208 & 68,2 & \\
\hline \multicolumn{8}{|c|}{ Consumo de marihuana } \\
\hline Sí & 7 & 4,5 & 15 & 10,1 & 22 & 7,2 & $P<0,05$ \\
\hline No & 150 & 95,5 & 133 & 89,9 & 283 & 92,8 & \\
\hline
\end{tabular}

NS: no significativo. Nota. Se aplicó el Test $\chi^{2}$.

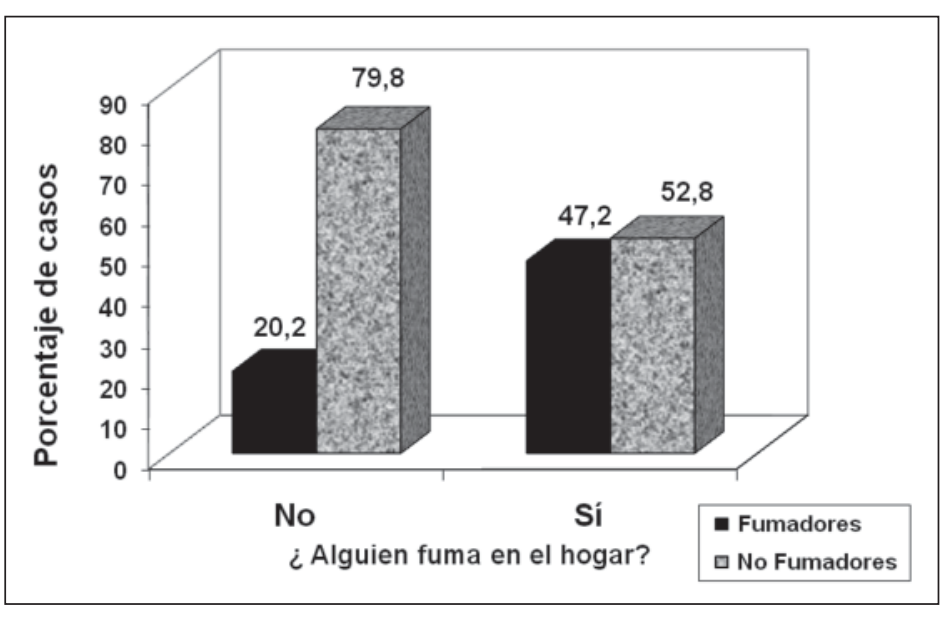

Figura 1. Comparación del porcentaje de estudiantes fumadores y no fumadores, según la presencia o ausencia de algún fumador en su hogar $(P<0,001)$. mensual de tabaco, alcohol, tabaco-alcohol. Por el contrario, la FAECS fue la que presentó las mayores prevalencias en cuanto al consumo de tabaco-alcohol y marihuana en el último mes y la FAI fue la que obtuvo la mayor prevalencia en cuanto al consumo de alcohol.

El comportamiento de los estudiantes de la FAS podría explicarse porque los estudiantes que postulan a carreras relacionadas con ésta área pudieran tener un mayor conocimiento e interés por estilos de vida saludables, en comparación con los estudiantes de otras carreras. Estos resultados coinciden con los reportados recientemente en estudiantes universitarios de Grecia, en los cuales un menor porcentaje de estudiantes de medicina consumía alcohol y tabaco, en comparación con estudiantes de otras facultades ${ }^{4}$. Por otra parte, los resultados del presente estudio también son similares a otros estudios realizados en Chile, que han encontrado que los estudiantes de medicina 
fuman menos que el resto de la juventud chilena y que los estudiantes de medicina de otros países, como Estados Unidos de Norteamérica ${ }^{7,11-15}$.

Las prevalencias del consumo de tabaco, alcohol, tabaco-alcohol y marihuana son mayores en los estudiantes que cursaban cuarto año, en comparación con lo de primero. Sin embargo, en cuanto a los gramos de alcohol consumidos por los estudiantes de primer año, fueron mayores que lo observado en los estudiantes de cuarto año, sin diferencias significativas. Este hecho podría explicarse por el tipo de bebida alcohólica consumida, ya que en primer año estarían consumiendo bebidas de mayor grado alcohólico. Estos resultados concuerdan con otros estudios que señalan que aquellos alumnos que cursaban primer año presentaron menor consumo de tabaco y alcohol ${ }^{5}$. También concuerdan con otro estudio realizado en universitarios de la carrera de Medicina en Colombia ${ }^{6}$. Los estudiantes de género femenino presentaron un menor consumo de tabaco, tabaco-alcohol, alcohol y marihuana, siendo significativas las diferencias para estas dos últimas.

Además es importante mencionar que, al comparar el consumo de tabaco de acuerdo al género y al año que cursaban los estudiantes, se observó que las mujeres de cuarto año son las que presentaron las mayores prevalencias de consumo, en comparación con los hombres del mismo curso, situación que coincide con otros estudios efectuados en Chile ${ }^{5}$. Sin embargo, al comparar las mujeres fumadoras de primero vs cuarto año, sólo se observaron diferencias significativas en la FAECS, en las cuales la prevalencia de consumo fue mayor en las que cursaban cuarto año.

Con respecto al consumo de tabaco, al comparar nuestros resultados con los del estudio CONACE 2008 (en personas que declararon que su actividad principal era estudiar en alguna universidad o instituto), la prevalencia del CONACE fue de 56,7 en hombres y $51,4 \%$ en mujeres ${ }^{3}$. En el presente estudio las prevalencias son menores, de $30 \%$ y $35,8 \%$ en mujeres y hombres, respectivamente. No obstante, estos resultados son similares a otro estudio realizado en universitarios chilenos ${ }^{5}$. Además se ha observado en trabajos realizados en países europeos, que los estudiantes de género femenino presentan un menor consumo de tabaco y alcohol ${ }^{4}$. La situación opuesta ha sido descrita en diversos estudios efectuados en universitarios chilenos, que señalan que la prevalencia de tabaquismo es mayor en las mujeres ${ }^{16,17}$.

Es destacable el hecho que, de los estudiantes que refieren que "Nadie fuma en el hogar", el 79,8\% corresponde a estudiantes "No fumadores". De esto se puede inferir que provenir de una familia no fumadora es un factor protector para los estudiantes, lo que coincide con otras investigaciones ${ }^{18,19}$.

Por otra parte, la restricción del consumo de tabaco y alcohol en la universidad, da cuenta de 39 y $38 \%$ de disminución del consumo de tabaco y alcohol, respectivamente. Esto podría indicar un efecto preventivo menor en los estudiantes universitarios.

Con respecto al consumo de alcohol en el último mes, la prevalencia del CONACE 2008 fue de 77,4 y $71,6 \%$, en hombres y mujeres, respectivamente (en personas que declararon que su actividad principal era estudiar en alguna universidad o instituto) y en el presente estudio la prevalencia obtenida fue de 73,6 y $52,9 \%$, en hombres y mujeres, respectivamente, observándose que la prevalencia en hombres encontrada en nuestro estudio es bastante similar a la del CONACE, sin embargo, la prevalencia en mujeres es bastante inferior. No obstante, en nuestro estudio se observó que el consumo de gramos de alcohol a la semana es muy similar entre estudiantes mujeres y hombres. Este hecho podría explicarse por el tipo de bebida alcohólica consumida, ya que las estudiantes estarían consumiendo bebidas de mayor grado alcohólico. Esto concuerda con los resultados de la Encuesta Nacional de Salud 2009, que señala que no existen diferencias en el consumo de gramos de alcohol entre hombres y mujeres de 15 a 24 años ${ }^{2}$. La situación opuesta se evidenció en otro estudio realizado en universitarios chilenos, encontrándose un mayor consumo de gramos de alcohol en los estudiantes hombres ${ }^{5}$.

En relación a las prevalencias en el consumo de marihuana durante el último mes, se obtuvo $10,1 \%$ y $4,5 \%$ en hombres y mujeres, respectivamente, valores que son bastante similares a los obtenidos en estudiantes de Medicina de la Pontificia Universidad Católica de Chile (5,1\%), sin diferencias significativas por género ni por cur$\mathrm{so}^{20}$. No obstante, estos valores son inferiores a las obtenidas por CONACE 2008, que reportó 22,3\% en hombres y $11,6 \%$ en mujeres. Estos valores podrían explicarse por una probable subdeclaración del consumo, en especial referente a drogas 
ilícitas, que podría subestimar la real magnitud del consumo de drogas ${ }^{3,17}$.

Nuestro estudio representa un aporte al diagnóstico de los estilos de vida de los estudiantes universitarios, que evidencian un aumento en las prevalencias en el consumo de tabaco, alcohol y marihuana, al comparar estudiantes de primer y cuarto año. Estos hallazgos invitan a implementar políticas tendientes a potenciar a la universidad como un entorno promotor de la salud de la comunidad educativa y de la sociedad en conjunto. Los resultados ponen de manifiesto la necesidad de promover estrategias en la comunidad educativa, a través de programas efectivos de educación en prevención del consumo de drogas, focalizados y diferenciados por facultad, curso y género. Por otra parte, nos parece necesario abordar un plan adecuado de comunicación para la salud, que incluya aspectos culturales, sociales, políticos e interculturales ${ }^{21-23}$.

Agradecimientos: Los autores agradecen a la Vicerrectoría de Investigación y Desarrollo, Departamento de Investigación (DI) de la Universidad de Chile, por el financiamiento del Proyecto SOC 09/09-1, gracias a lo cual ha sido posible comenzar esta línea de investigación emergente. Los datos del presente manuscrito forman parte de la tesis de Gladys Morales Illanes, como uno de los requisitos con los cuales obtuvo el grado académico de Magíster en Nutrición y Alimentos con mención en Promoción de la Salud y Prevención de Enfermedades Crónicas Asociadas a la Nutrición, en el Instituto de Nutrición y Tecnología de los Alimentos (INTA), de la Universidad de Chile; codirigida por los profesores Mg. Daniza Ivanovic y Dr. Carlos del Valle Rojas. Además se agradece a la Srta. Cecilia Retamal y a la Srta. Elizabeth Loaiza por su valioso aporte en la presente investigación.

\section{Referencias}

1. Organización Mundial de la Salud (OMS). Informe sobre la salud en el mundo 2002. Reducir los riesgos y promover una vida sana. Ginebra.

2. Ministerio de Salud. Encuesta Nacional de Salud 2009. Disponible en: http://www.redsalud.gov.cl/portal/ docs/page/minsalcl/g_home/submenu_portada_2011/ ens2010.pdf [consultado el 01 de junio 2010].

3. Consejo Nacional para Estupefacientes (CONACE).
Octavo Estudio Nacional de drogas en población general de Chile. 2008. Comunicación personal con Sr. Esteban Pizarro, 13 de junio 2010.

4. Tirodimos I, Georgouvia I, Savvala TN, Karanika E, Noukari D. Healthy lifestyle habits among Greek university students: differences by sex and faculty of study. East Mediterr Health J 2009; 15 (3): 722-8.

5. Durán S, Castillo M, Vío F. Diferencias en la calidad de vida de estudiantes universitarios de diferente año de ingreso del campus Antumapu. Rev Chil Nutr 2009; 36 (3): 200-9.

6. Alba LH. Perfil de riesgo en estudiantes de Medicina de la Pontificia Universidad Javeriana Univ Med Bogotá (Colombia) 2009; 50 (2): 143-55.

7. Romero MI, Santander J, Hitschfeld M, Labbé M, Zamora V. Consumo de tabaco y alcohol entre los estudiantes de medicina de la Pontificia Universidad Católica de Chile. Rev Med Chile 2009; 137: 361-8.

8. Muñoz M, Cabieses B. Universidades y promoción de la salud: ¿cómo alcanzar el punto de encuentro. Rev Panam Salud Pública 2008; 24 (2g): 139-46.

9. Declaración de Helsinki de la Asociación Médica Mundial (2008). Disponible en: http://declaraciondehelsinki. blogspot.com[consultado el 06 de junio 2010].

10. Guilford JP, Fruchter B. Estadística Aplicada a la Psicología y a la Educación. México: McGraw Hill. 1984.

11. Pickard M, Bates L, Dorian M, Greig H, Saint D. Alcohol and drug use in second-year medical students at the University of Leeds. Medical Education 2000; 34: 148-50.

12. Newbury-Birch D, White M, Kamali F. Factors influencing alcohol and illicit drug use amongst medical students. Drug and Alcohol Dependence 2000; 59: 125-30.

13. Mangus R, Hawkins C, Miller M. Tobacco and alcohol use among 1996 medical school graduates. JAMA 1998; 280: 1192-3.

14. Newbury-Birch D, Walshaw D, Kamali F. Drink and drugs: From medical students to doctors. Drug and Alcohol Dependence 2001; 64: 265-70.

15. Mcauliffe W, Rohman M, Breer P, Wyshak G, Santangelo S, Magnuson E. Alcohol use and abuse in random samples of physicians and medical students. Am J Public Health 1991; 81: 177-82.

16. Mc Coll P, Amador M, Aros J, Lastra A, Pizarro C. Prevalencia de factores de riesgo de enfermedades crónicas no transmisibles en estudiantes de medicina de la Universidad de Valparaíso Rev Chil Pediatr 2002; 73 (5): 478-82.

17. Rodríguez J, Hernández E, Fernández AM. A gender comparison of legal and illicit drug consumption. Rev Med Chile 2007; 135: 449-56.

18. García R, Varona P, Hernández M, Chang M, Bonet M, 
García M. Rev Cubana Hig Epidemiol 2008; 46 (3) 1-9. Disponible en: www.scielo.sld.cu [consultado el 01 de junio 2010].

19. González H, Berger K. Consumo de tabaco en adolescentes: factores de riesgo y factores protectores. Cienc enferm 2002; 8(2): 27-35. Disponible en:www.scielo.cl/ scielo [consultado el 01 de junio 2010].

20. Romero M, Santander J, Hitschfeld M, Labbé M, Zamora V. Consumo de sustancias ilícitas y psicotrópicos entre los estudiantes de medicina de la Pontificia Universidad Católica de Chile. Rev Med Chile 2009; 137: 459-65.

21. Del Valle, C. Comunicar la salud. Entre la equidad y la diferencia. T. 2002. Temuco: Ediciones Universidad de La Frontera.

22. Del Valle C, Cofré E. “Formación en salud intercultural: fundamentos socioculturales y proyecciones educomunicativas". En Hidalgo, J. (Coord.): Signo Vital: Comunicación estratégica para la promoción de la salud. 2011. Huixquilucan, Estado de México, Centro de Investigación para la Comunicación Aplicada de la Universidad Anáhuac México Norte. [En prensa].

23. Del Valle C, Cofré E. Comunicar la salud en contexto: Crítica y praxis educomunicativa, Tempus Actas de Saúde Colectiva 2009; 3 (1): 38-48. 